\title{
AN APPLICATION OF THE METHOD OF MULTI- DIMENSIONAL SCALING TO PERCEPTION OF SIMILARITY OR DIFFERENCE IN COLORS*
}

\author{
TAROW INDOW AND TEIKO SHIOSE \\ Keio University
}

\section{INTRODUCTION}

Recently Torgerson made animportant contribution to psychophysical scaling methods. So far several kinds of methods have been devised for assigning rational numerical values to stimuli or items in regard to an attribute which is not describable in physical terms. We encounter the necessity of this kind of scaling especially in the field of attitude measurement, and the method of paired comparisons and the method of successive intervals are to be mentioned as representative ones for such purposes. Different methods involve different experimental procedures for obtaining subjects' responses

* The writers are deeply indebted to Educational Testing Service, Princeton, for the kindness of making available its Research Bulletins without which this study could not have come to birth. Dr. Yokoyama kindly read through the manuscript, gave valuable suggestions and also criticisms concerning English. We wish to thank Mr. Murakami and the staff of Color Section of Japan Electronic Instruments Co.Ltd., for the interest they took in this experiment.

${ }^{1}$ In his Psychometrika article (1) the part concerning his empirical investigation was omitted, but it was fully described in the Research Bulletin (2). The writers are greatly indebted to the latter. to stimuli or items presented and also different sets of assumptions behind their formulae to calculate scale values. In any case, there is a restriction to the application of these methods because they presuppose a single dimensionality in the domain to be scaled. In order to apply these methods successfully, the assumption has to be accepted that all stimuli or items be arranged adequately along a single continuum and if the assumption does not fit the data we are obliged to abandon the attempt of bringing a scale into the domain or to analyze the domain further into smaller parts until the assumption is met.

This restriction was removed, however, in the method of multi-dimensional scaling proposed by Torgerson in which the dimensionality was determined from the data themselves. As an empirical verification he applied the method to the perception of similarity or difference between pairs of red colors which differed in saturation and brightness, hence the dimensions of this domain in question could be anticipated to be two. ${ }^{1}$ The method essentially consisted in generalizing the method of paired comparisons and in adopting techniques of the factor analysis. It is interesting to 
note that the procedures like these which have been primarily used in the field of attitude measurement and intelligence find an excellent application also to the problem of perception.

A distinction has to be made, on the other hand, between Torgerson's method and usual psychophysical procedures which are frequently employed when quantification of the sensory magnitude is aimed at $(\mathbf{3}, \mathbf{4})$. For example, scales of loudness or pitch have been successfully developed by the method of equation, bisection and by the juxtaposition of jnd's, etc. $(5,6)$. While these methods can be applied only when subjects know what is meant by the attribute in question, e.g., loudness or pitch, no previously defined continuum is required in the method proposed by Torgerson and it becomes possible to study the domain where neither dimensions themselves nor the number of relevant dimensions are apparent. Besides, since each dimension of a particular domain has to be scaled separately in the traditional psychophysical methods, no knowledge of the relation between different dimensions is obtained. This impossibility of handling multi-dimensional variations will be fatal if such problems are inquired as similarity or difference which may possibly be a complex function of more than two attributes. As we shall see presently, scaling of similarity or difference in

3 The abbreviation of Commission Internationale de l'Eclairage. the area of color plays an important rôle in developing the color space and in such an area the satisfactory result may not be expected unless a method becomes available, which enables one to determine scale values corresponding to each stimulus on all relevant dimensions. As to similarity or difference in the multi-dimensional domain, Torgerson's method would provide us with informations which would be unavailable with the usual procedures.

The system of color specification can be physically or psychologically developed. While the $\mathrm{CIE}^{2}$ system, the well known chromaticity diagram, is defind entirely upon physical measurements of colored lights, colors can also be set in spatial order in accordance with their perceptual appearance and the Munsell system is a characteristic example of this type of color space. In any case, it is desirable that colors be represented as points lying in a space in such a way that the magnitude of distance between any two points is proportional to the degree of perceptual difference between two colors corresponding to these points. Not only colors appearing similar should be spaced near and ones appearing different far apart but also the quantitative proportionality between the distance in the space and the difference in the appearance should exactly hold through the whole range of colors which vary in hue, brightness and saturation. In other words, a given linear extent in the space, wherever it may be and in whatever direction it 
may lie, should represent an equal sense distance. The spatial arrangement of colors which satisfies this requirement and which therefore enables one to tell to what degree any two colors differ in perception is called the uniform color scale and generally abbreviated as UCS (7).

In the present article we will not go into the complicated problem of developing the UCS which has been attempted by the spectrophotometric measurements; we wish only to examine the psychological aspect of the Munsell system. Some regard the Munsell system as the "empirical ideal", the best available approximation to the UCS (8). It is generally agreed, however, that the Munsell solid approximates the UCS in regard to its three dimensions taken separately, but not necessarily in regard to its three dimensions taken collectively $(9)$. Colors in the the Munsell solid are so arranged that equal linear distances along the vertical brightness axis or radial saturation axis or equal angular displacements along the hue circle represent equal steps in sensation with respect to each color attribute. Thus, for example, as to two colors $a$ and $b$ corresponding to two points in the Munsell system, (cf. Fig.1), we learn that they differ in saturation by the amount corresponding to $\left(X_{a}-X_{b}\right)$ and in brightness by the amount corresponding to $\left(Y_{a}-Y_{b}\right)$, nevertheless it is not clear what the linear distance between the two points, the solid line in Fig. 1 indicates. For spacing to be valid and consistent for multi-di mensional variations of color it is not sufficient, therefore, that each dimension is defined in terms of the unit which is proved to be appropriate when comparison is made between colors in regard to one particular attribute with other attributes held constant. Comparison between colors as wholes is of importance and what definition is to be made as to the distances that represent such differences should be determined experimentally. That the attributes be represented by the mutually orthogonal reference axes and that the geometry which defines the distances be Euclidean in nature are not at all self-evident. For example, the formula proposed by Nickerson (10) for evaluating multidimensional color differences led to a

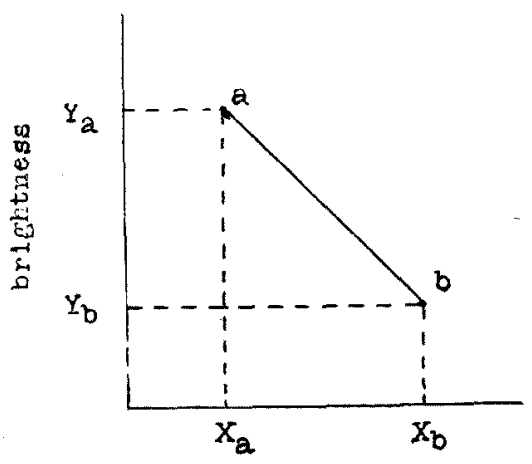

Fig. 1 Saturation

non-Euclidean geometry. The application of the multi-dimensional scaling would seem to throw light upon a geometry according to which colors are set into the UCS.

The possibility of achieving the UCS 
arrangement of colors in the Euclidean space of three dimensions is also a necessary condition for the color pyramid to be more than a mere schematic representation. If it becomes clear on the basis of experimental facts that this condition is not satisfied, the Munsell system or the color pyramid would be misleading unless it is understood as a mere schema which lacks appropriate metrics to multi-dimensional color differences.

\section{Purpose}

The purpose of this study was similar to that of Torgerson's empirical application mentioned above. Using as stimuli, nine Munsell colors and adopting the multi-dimensional scaling method, we sought to determine (a) to what extent the nine colors could be approximated by a configuration of points lying in two dimensional Euclidean space, (b) whether there were two mutually orthogonal axes which would represent brightness and saturation respectively, and (c) how the configuration of points would compare with the Munsell system. In this study neither development of the UCS nor standardization of any kind were intended. If the intention has been of primary concern, more careful specification of color samples, more careful control of illumination and more extensive selection of stimuli would have been indispensable.

The multi-dimensional scaling method involves three basic steps. In the first step, a scale of comparative distances regarding similarity between all pairs of stimuli is obtained. The second step involves an estimation of an additive constant and conversion of the comparative distance into absolute ones using this estimate. The importance of this step will be discussed later. In the third step, the dimensionality of the psychological space necessary to account for these absolute distances is determined, and the projections of the stimuli on axes of this space are obtained.

Torgerson applied the method of paired comparisons to responses of the subjects obtained by the complete method of trials in the first step of the scaling procedure. As to the colors $k, i$ and $j$ presented in triads, the subjects were required to judge whether color $k$ looked more like color $i$ or color $j$ and the judgments of all subjects, 40 in number, were pooled to give the proportion of the subjects who judged color $k$ more similar to color $j$ than color $i,{ }_{k} P_{l}$. In order to obtain the comparative distance $H_{j k}$ between color $j$ and color $k$ for all possible pairs of nine colors used, Torgerson derived from ${ }_{k} P_{i j}$ the difference between the comparative differences, ${ }_{k} X_{i}=\left(H_{k k}-\right.$ $H_{k j}$ ), on a set of assumptions which were essentially the same as those underlying Thurston's Case V in the law of comparative judgements (11). Then the scale of $H_{J k}$ was developed by the least square method which Torgerson devised. It should be re- 
bered, however, that the judgments of all subjects were far from complete agreement, viz., color $k$ looked more like color $i$ to some subjects and more like color $j$ to others and it was on the basis of this disagreement that Torgerson developed the scale of comparative distances. Had the judgments of all subjects been in complete accordance, it would have been impossible to develop the scale because ${ }_{k} P_{i j}$ made no contribution toward the scaling in his procedure if it turned out 1 or $0 .{ }_{k} X_{i j}$ would be $+\infty$ or $-\infty$ in such cases. Therefore, it is clear that the comparative distances $H_{J k}$ thus determined is defined only with respect to the collective data of the subjects in which individual differences can by no means be neglected. As a matter of course, $H_{j k}$ may be regarded as a kind of average defined on a set of assumptions over the subjects, but as to individual values to be averaged no information is provided in this procedure. There is no way of checking whether individual values are randomly distributed around the average and it will be difficult to appreciate adequately the results derived from this procedure if perception of similarity by each individual comes into question. Torgerson obtained, analyzing $H_{j k}$ thus scaled, the final results which agreed surprisingly well with the Munsell

3 It was impossible to obtain the exact colored papers which were needed. So nine colors which we actually used in our experiment were somewhat different also in hue as shown in Table 1. scales, so he concluded that saturation and brightness could be represented adequately by a Euclidean model. For the reason mentioned above, however, his conclusion applies to responses of the subjects as a whole but not necessarily to those of each subject. It is not decided whether the subjects perceive colors more or less in the same way as indicated in his conclusion, or there are some subjects who perceive colors in strikingly different ways from others and thus his conclusion is warranted only with respect to averages. Individual differences in this case might be qualitative as well as quantitative in nature.

To be free from this criticism, we tried to obtain separate results for each subject by the use of the multi-dimensional scaling for individual measurements. Namely, in the first step of the scaling procedure, the comparative distances between all pairs of colors were determined from the data of a single individual by the method which will be described in the following section.

\section{EXPERIMENTAL PROCEDURE}

Nine colors similar to those used by Torgerson were chosen as stimuli. They were of the same red hue ${ }^{3}$ but different from each other in brightness and saturation. The photometrically determined Munsell notations of these colored papers are listed in Table. 1, where the first term indicates hue, the 
second value (brightness) and the third chroma (saturation), ${ }^{4}$

Table. 1

The Photometrically Determined Munsell Notations of the Colored Papers.

\begin{tabular}{c|ccc}
\hline & hue & value & chroma \\
\hline 1 & $8.0 \mathrm{R}$ & 7.1 & $5.0 \quad$ (*) \\
2 & $5.0 \mathrm{R}$ & 6.3 & 5.9 \\
3 & $5.0 \mathrm{R}$ & 6.1 & 9.6 \\
4 & $4.8 \mathrm{R}$ & 5.1 & 4.7 \\
5 & $5.0 \mathrm{R}$ & 5.0 & 9.4 \\
6 & $5.9 \mathrm{R}$ & 5.1 & 12.0 \\
7 & $5.5 \mathrm{R}$ & 4.1 & 6.2 \\
8 & $5.3 \mathrm{R}$ & 4.2 & 9.3 \\
9 & $7.1 \mathrm{R}$ & 3.3 & 3.5 \\
$*$ & The terms in this row are questioned. \\
This colored paper was inappropriate to \\
be specified photometrically.
\end{tabular}

Each of these colored papers was cut in the form of a rectangle, $2.5 \mathrm{~cm} \times$ $7.5 \mathrm{~cm}$ and was pasted on the upper part of a thick cardboard, $2.5 \mathrm{~cm} \times$ $10.5 \mathrm{~cm}$, the remaining part of it was covered with neutral gray paper. In order to keep the card unblemished, the cardboard was always handled by the gray part. Four series of stimuli were prepared and they were used alternately.

The subject was seated in front of a table facing a screen placed $50 \mathrm{~cm}$. away from him, which, having two wings on both sides, occupied most of his visual field. The screen and the entire table were covered with light gray

4 The colored papers were obtained from the Nippon Color Research Institute. paper. In the center of the screen a sheet of neutral gray paper, $40 \mathrm{~cm} \times$ $30 \mathrm{~cm}$ was mounted, upon which the subject was to gaze when the stimulus was not presented.

On the center of the table, between the screen and the subject, a wooden board $30 \mathrm{~cm}$ wide, $10 \mathrm{~cm}$ long, $4.5 \mathrm{~cm}$ thick at one end and $3 \mathrm{~cm}$ thick at the other end, was placed in such a way as to make its upper surface slope down toward the subject. This board was covered also with neutral gray paper. Three colors were presented on this board for comparison (vide infra). The light was furnished by two Fluores cent 20-watt daylight lamps and two Mazda 60-watt daylight lamps which were arranged in three rows just $80 \mathrm{~cm}$ above the stimulus field as advised by the Mazda Laboratory. All extraneous light was excluded by a black curtain which surrounded the table, the subject and the experimenter. The illumination of the field was approximately 200 luces.

Three subjects, In, $\mathrm{S}$ and $\mathrm{H}$, took part in this experiment. $\mathrm{S}$ and $\mathrm{H}$ were graduate students in psychology and In was the senior author. The junior author worked as the experimenter. S and $\mathrm{H}$ had no knowledge of the purpose of this experiment. All subjects were normal in color vision and none have had special training in the observation of color except in the ordinary laboratory course in experimental psychology. The experiment was conducted individually. The following instructions 
were given to the subject at the beginning of each experiment.

"You will be sitting at this table and looking at the gray paper mounted in the center of the screen. I will place a card on each side of the board and another in the center. You will see three cards more or less in different colors. First you decide whether the color in the center looks more like the right or the left one. Then you displace the one in the center towards the more similar one in accordance with the degree of the difference in similarity between the two pairs of colors, the center and the right, the center and the left. For instance, if you see three color looking almost alike but decide the center looks more nearly the same as the right, displace the center slightly to the right, or if you see the center looking quite different from the other two but decide the center looks a litcle more like the right, displace the center slightly to the right. If you see the center looking much like the right, displace the center a good deal to the right. Or if you cannot decide whether the center looks more like the right or the left because two pairs of colors, the center and the right, the center and the left, look similar exactly in the same degree or look different exactly in the same degree, then do not displace the center to either side. You are free to displace the center to the right or to the left until you are satisfied with your final result. Remember, what I want here is not an analytical judgment but a total impression that this color looks mo:e like that one. The impression of similarity may have different nuance from pair to pair but there is no need to worry about it. While I arrange cards for the next presentation please keep your eyes on the gray paper mounted in the center of the screen."

The experimenter, sitting behind the wing of the screen and to the left of the subject, placed on the table the board on which the stimuli were arranged. While the subject was performing the task assigned, the experimenter recorded the preceding judgment and arranged stimuli on another board for the next presentation.

As described in the instructions, a triad of colors $i, j$ and $k$ was presented in a row on the board, $k$ occupying the middle position between $i$ and $j$ whose distance was fixed at $24 \mathrm{~cm}$. The subject had to express the difference in the degree of similarity between $i$ and $k$ and that between $j$ and $k$, by displacing $k$ from the midpoint of the board. The difference between comparative distances, ${ }_{k} X_{i j}=\left(H_{i k}-H_{k j}\right)$, was quantified in this way. The subject was to transform the magnitude of the perceived difference to that of spatial distance of his own accord. In order to establish a frame of reference concerning this transformation, each subject trained himself about 200 times in the preliminary experiment with gray stimuli and with the same color stimuli as used in the main experiment. During this preliminary experiment, no remarks were given by the experimenter with respect to judgments of the subject. This procedure should not be confused with placing $k$ so as to directly represent on the board $H_{i k}$ and $H_{k j}$ in accordance with the ratio of similarity between $i$ and $k$ to that between $j$ and $k$. What was to be expressed was neither $H_{i k}, H_{k}$, nor $H_{i k} / H_{k}$, but it was $\left(H_{i k}-H_{k j}\right)$. Only the difference was represented but not its constituents. In other words, it was immaterial how the distance between $i$ and $k$ and that between $j$ and $k$ came out on the board 
as a consequence of the displacement of $k$. These distances could by no means be regarded as measures of $H_{\varepsilon k}$ and $H_{k}$, because the meaning of these distances necessarily varied from triad to triad. The same distances might sometimes correspond to similarity between two colors looking alike and sometimes to that between two colors looking different. Our concern was only the position of stimulus $k$ after the displacement measured in centimetres from the midpoint and it was recorded as ${ }_{k} X_{i j}$, an empirical value for ${ }_{k} X_{i j}=\left(H_{i k}-H_{k \jmath}\right)$.

The main experiment, which was carried out individually, consisted of 10 sittings which took about an hour each. The observation was made after about 5 minute adaptation to the illumination in each sitting. Eighty four triads were formed with nine colors, in each triad there were six different ways of arranging the items, the total number of stimuli was $84 \times 6=504$. They were presented in random order. The experiment was repeated two times making the total number of presentation 1008 . However, since out of the 504 stimuli $i-k-j$ and $j-k-i$ were treated as equivalent in the calculation of the results, we may say that 252 stimuli were presented four times in the entire experiment.

All subjects came to realize that the task of judging was not as difficult as has been expected, but no one had confidence as to whether the judgment was conducted exactly as required by the instructions. Though they were required to express the same difference in the impressions by the same spatial distance no matter whether it was the difference in the degree of similarity or of dissimilarity, it was found more difficult to differentiate the degree of difference in dissimilarity than to differentiate the degree of difference in similarity. The "end effect" could not be avoided, i.e., there was a tendency that the maximum displacement, $12 \mathrm{~cm}$. which should be assigned only to the maximum possible dissimilarity, was also given to dissimilarities of lesser degrees.

\section{ANALYTICAL PROCEDURE}

In the present experiment, ${ }_{c} X_{i j}=$ $\left(H_{i k}-H_{k j}\right)$ was directly quantified as mentioned above. No additional assumptions were necessary except that the subject made judgments as required in the instructions and was able to assign the same displacement to the same degree of difference in similarity or dissimilarity. Whether or not the assumption was acceptable would be decided by final results. The rest of the calculating processes in the analysis was carried after those of Togerson's.

As four independent estimates of each ${ }_{6} X_{i j}$ were in good agreement, they were simply averaged to obtain an empirical value ${ }_{k} X_{i j}$. From nine matrices, whose elements were ${ }_{k} X_{t,}$, the estimates for the comparative distances $H_{j k}$ between $j$ and $k$ for all possible pairs of nine colors were determined by the equation : 
$h_{j k}=1 / 2\left({ }_{g} X_{h}+X_{\cdot g}+{ }_{h} X_{\cdot g}+X_{\cdot h}\right)$,

where

$$
\begin{aligned}
& { }_{g} X_{\cdot_{h}}=\frac{1}{n-1} \sum_{i} X_{i h} \\
& { }_{h} X_{\cdot_{g}}=\frac{1}{n-1} \sum_{i} X_{i h} \\
& X_{\cdot g}=\frac{1}{n(n-1)} \sum_{i h} \sum_{h} X_{i j} \\
& . X_{\cdot h}=\frac{1}{n(n-1)} \sum_{i g} \sum_{g} X_{i h} \\
& g, h, i, j, k=1,2, \ldots \ldots \ldots n .
\end{aligned}
$$

This is the least square solution given by Torgerson in minimizing the follow. ing function :

$$
2 F=\sum_{k} \sum_{j} \sum_{i}\left[k X_{i j}-\left(H_{i k}-H_{k j}\right)\right]^{2} .
$$

The next step in the analysis was estimation of an additive constant $c$ by which the comparative distances, $h_{j k}$, were converted into absolute ones, $\boldsymbol{d}_{j k}$. Necessity of this conversion was clearly illustrated by Torgerson. In order to see whether a set of interpoint distances could be considered to be the distances between points lying in a real Euclidean space, the interpoint distances had to be absolute ones possessing the true zero point, $d_{j J}=0$. On the contrary, the scale of comparative distances was defined upon an arbitrary zero and $h_{j}$ remained undetermined. In equation (3) :

$$
d_{j k}=h_{j k}+c \text {, }
$$

the choice of a particular value of $c$ would have an important influence upon the subsequent multi-dimensional mapping of the colors, viz., upon the nature and the dimensionality of the color space. The criterion of the choice proposed by Torgerson was to select such a value that allow the colors to be fitted by a real Euclidean space of the smallest possible dimensionality. Although an analytical solution for the additive constant was described in (12, 13), these papers were not available when we analyzed our data, so that we followed the procedures recommended by Torgerson which will be described later.

From the distance matrix $D$ consisting of $d_{j k}$, the scalar product of vectors to points $j$ and $k$ from the centroid of the points, $b_{j r_{i}}{ }$, was determined by the equation :

$$
\begin{array}{r}
b_{j k}^{*}=b_{j k}-\frac{1}{n} \sum_{j} b_{j k}-\frac{1}{n} \sum_{k} b_{j k} \\
+\frac{1}{n^{2}} \sum_{j} \sum_{k} b_{j k},
\end{array}
$$

where $b_{j k}$ was given by

$$
b_{j k}=1 / 2\left(d^{2}{ }_{1 j}+d^{2}{ }_{1 k}-d^{2}{ }_{j k}\right) \text {. }
$$

Once the matrix $B^{*}$ whose elements are $b_{J k}{ }^{*}$ is obtained, it becomes possible to determine the dimensionality of the space necessary to account for the distance matrix $D$ and to obtain the projections of points on axes of this space, because if the matrix $B^{*}$ is positive semi-definite, that means, if $r$ latent roots of $B^{*}$ have large positive values and the remaining $(n-r)$ are zero disregarding errors, then it can be written as follows :

$$
B^{*}=A A^{\prime},
$$

where $A=a_{j m}$ is the matrix of the projections of points $j$ on axes $m$ of the arbitrary coordinate system $(m=$ $1,2, \ldots \ldots \ldots . . . r)$ with origin in the centroid. 
In this way, the dimensionality of the space $(r)$ and the configuration of the points $\left(a_{j m}\right)$ will be determined. It is noteworthy that the colors can not be considered as lying in a real Euclidean space unless $B^{*}$ is semi-definite. We can also see from the data themselves whether this condition is fulfilled or not. Besides, the configuration of the points given by $a_{j m}$ will enable us to introduce into the space the new coordinate system which is not arbitrary but psychologically meaningful. In other words, each axis will represent an attribute. This is achieved by the rotation of axes as follows:

$$
V=A A,
$$

where $V=v_{J m}$ is the matrix of the projections of points $j$ on axes $m$ of the new coordinate system $(m=1,2$, $\ldots \ldots \ldots . . . r)$ with origin in the centroid and $A$ is the transformation matrix.

We owe the principal idea of this analysis to Young and Householder (14) but Torgerson modified the procedures applicable to fallible data. It is his idea to take the centoid as the origin for the sake of obtaining an unique solution. There is no special difficulty in factoring $B^{*}$ in the equation (6) and in obtaining the meaningful set of axes because these procedures are routines in the factor analysis. It is to be mentioned that in contrast to the factor analysis we can get rid of the difficult problem of estimating the principal diagonal $b_{j}$ of $B^{*}$ as they are directly given in the data.
To estimate the value of $c$ in the equation (3), we followed Torgerson's procedures. He recommended two methods as follows:

(a) Obtain the $n(n-1)(n-2) / 6$

values of $z$ :

$$
\begin{aligned}
z=h_{i \jmath}+h_{j k}-h_{i k} \\
\quad\left(h_{i k}>h_{i j}>h_{j k}\right)
\end{aligned}
$$

assuming in turn that each set of three colors lie in a line if the four sets of three of any four points give about the same "highest" value of $z$ in a consistent manner, it can be concluded that the four points are in a one-dimensional subspace and this value of $z$ will be accepted as an estimate of $c$. (b) Pick up any three stimuli which are expected to be linear from their positions in the Munsell system, and see whether they give about the same value of $z$ in (8). If they do, this value will be accepted as an estimate of $c$. At first, the data of the subject $S$ were analyzed by these procedures and $c=48.00$ was obtained by (a) and $c=38.66$ by (b). For these two values of $c$ the remaining matrices, $D, B$ and $A$ were constructed. As was the case with the study of Torgerson, it became apparent that the precise value of $c$ was not too important with respect to the spacing of the points since configurations which were obtained by two sets of $a_{j m}$ resulting from the two estimates coincided very closely. As to the subject $S$, therefore, only the result based on $c=38.66$ will be considered in the following discussion. In the analysis of the data of the other 
subject, the value of $c$ was determined respectively by taking into consideration two estimates given by (a) and (b) with the following results : $c=60.00$ for the subject In, and $c=110.00$ for the subject $H$. The exact values were 54.07 by (a) and 63.80 by (b) in the former and 118.91 by (a) and 100.89 by (b) in the latter.

\section{RESULTS}

The matrices $B^{*}$ for all subjects are shown in Table 2 to 4 . Each of these matrices was then factored by the centroid method to give the matrix $A=$ $a_{j m} \quad(m=1,2, \ldots \ldots \ldots . r)$, which determined the configuration of the colors in the $r$ dimensional space. As shown in Table 5 to 7 , the factoring was continued until three factors were extracted in each case $(r=3)$. In the following discussion, however, only the first and the second factors are taken into consideration $(r=2)$. The reason for dis-

Table. 2

Matrix $B^{*}$ TroR thr SUbJfet $\mathrm{S}$

\begin{tabular}{|c|c|c|c|c|c|c|c|c|c|}
\hline & 1 & 2 & 3 & 4 & 5 & 6 & 7 & 8 & 9 \\
\hline 1 & 1308.55 & 1016.77 & 487.21 & 491.15 & -565.20 & -629.25 & -930.79 & 797.52 & -380.94 \\
\hline 2 & 1016.77 & 733.69 & 521. 44 & 508.84 & -314.50 & -423.45 & -639.77 & 783.67 & -619.34 \\
\hline 3 & 487.21 & 521.44 & 398.11 & -93.19 & 208.52 & 239.80 & -556.28 & -293.72 & -911.87 \\
\hline 4 & 491.15 & 508.84 & -93.19 & 555.25 & -352.08 & -809.89 & 127.11 & -667.45 & 240.20 \\
\hline 5 & -565.20 & -314.50 & 208.52 & -352.08 & 292.16 & 487.20 & 205.24 & 481.28 & -442.63 \\
\hline 6 & -629.25 & -423.45 & 239.80 & -809.89 & 487.20 & 897.45 & -129.23 & 706.10 & -338.79 \\
\hline 7 & -930.79 & -639.77 & -556.28 & 127.11 & 205.24 & -129.23 & 588.79 & 539.69 & 795. 22 \\
\hline 8 & -797.52 & -783.67 & -293.72 & -667.45 & 481.28 & 706.10 & 539.69 & 800.35 & 14.92 \\
\hline 9 & -380.94 & -619.34 & $-911 . \dot{87}$ & 240.20 & -442.63 & -338.79 & 795.22 & 14.92 & 1643.25 \\
\hline
\end{tabular}

Table. 3

Matrix $B^{*}$ for the Subingte IN.

\begin{tabular}{r|rrrrrrrrr}
\hline & \multicolumn{1}{|c}{1} & \multicolumn{1}{c}{2} & \multicolumn{2}{c}{3} & \multicolumn{1}{c}{4} & \multicolumn{1}{c}{5} & \multicolumn{1}{c}{6} & \multicolumn{1}{c}{7} & \multicolumn{1}{c}{8} \\
\hline 1 & 2981.98 & 2409.56 & 1515.17 & 647.76 & -745.62 & -998.35 & -1708.40 & -2092.72 & -2009.40 \\
2 & 2409.56 & 1841.02 & 1506.72 & 668.32 & -230.51 & -924.97 & -1509.02 & -1622.06 & -2139.05 \\
3 & 1515.17 & 1506.72 & 1317.86 & -126.28 & 230.65 & 549.04 & -1520.39 & -1153.09 & -2319.68 \\
4 & 647.76 & 668.32 & -126.28 & 275.14 & -325.75 & -2022.10 & 282.75 & -754.07 & 1354.25 \\
5 & -745.62 & -230.51 & 230.65 & -325.75 & 464.65 & 1119.55 & 168.00 & 618.29 & -1299.26 \\
6 & -998.35 & -924.97 & 549.04 & -2022.10 & 1119.55 & 2763.56 & -541.81 & 1393.91 & -1338.81 \\
7 & -1708.40 & -1509.02 & -1520.39 & 282.75 & 168.00 & -541.81 & 1394.58 & 1176.60 & 2257.68 \\
8 & -2092.72 & -1622.06 & -1153.09 & -754.07 & 618.29 & 1393.91 & 1176.60 & 1399.93 & 1033.22 \\
9 & -2009.40 & -2139.05 & -2319.68 & 1354.25 & -1299.26 & -1338.81 & 2257.68 & 1033.22 & 4461.05
\end{tabular}


Table. 4

Matrix $B^{*}$ for the Stebinct H.

\begin{tabular}{r|rrrrrrrrr}
\hline & \multicolumn{1}{|c}{1} & 2 & 3 & \multicolumn{1}{c}{4} & \multicolumn{1}{c}{5} & \multicolumn{1}{c}{6} & \multicolumn{1}{c}{7} & \multicolumn{1}{c}{8} & \multicolumn{1}{c}{9} \\
\hline 1 & 8256.42 & 6277.68 & 3250.18 & 2890.80 & -3340.50 & -4128.94 & -5651.06 & -4878.70 & -2675.86 \\
$\mathbf{2}$ & 6277.68 & 4562.35 & 3279.87 & 3401.50 & -2143.03 & -3098.02 & -4543.72 & -4625.13 & -3111.46 \\
3 & 3250.18 & 3279.87 & 2762.47 & -473.22 & 1087.35 & 587.94 & -3448.72 & -1799.58 & -5246.23 \\
4 & 2890.80 & 3401.50 & -473.22 & 3781.99 & -1609.15 & -6573.12 & 1666.21 & -5929.81 & 2844.83 \\
5 & -3340.50 & -2143.03 & 1087.35 & -1609.15 & 2273.40 & 3723.31 & 1675.87 & 3555.80 & -5223.05 \\
6 & -4128.94 & -3098.02 & 587.94 & -6573.12 & 3723.31 & 9836.73 & -2556.76 & 6648.52 & -4439.65 \\
7 & -5651.06 & -4543.72 & -3448.72 & 1666.21 & 1675.87 & -2556.76 & 4209.85 & 2555.72 & 6092.63 \\
8 & -4878.70 & -4625.13 & -1799.58 & -5929.81 & 3555.80 & 6648.52 & 2555.72 & 7038.74 & -2565.51 \\
9 & -2675.86 & -3111.46 & -5246.23 & 2844.83 & -5223.05 & -4439.65 & 6092.63 & -2565.51 & 14324.30
\end{tabular}

Table. 5

The Extracted Factors for the SubJect $\mathrm{S}$.

\begin{tabular}{r|rrr}
\hline & \multicolumn{1}{|c}{$a_{1}$} & \multicolumn{1}{c}{$a_{2}$} & \multicolumn{1}{c}{$a_{3}$} \\
\hline 1 & 35.10 & -1.17 & -13.55 \\
2 & 29.54 & 1.13 & 0.31 \\
3 & 13.95 & 18.14 & 0.87 \\
4 & 15.53 & -18.09 & 12.39 \\
5 & -10.87 & 15.09 & 8.29 \\
6 & -17.24 & 25.04 & -8.46 \\
7 & -21.24 & -12.14 & 10.66 \\
8 & -27.01 & 8.75 & -1.02 \\
9 & -17.76 & -36.75 & -9.49 \\
$\sum_{j} a_{j m}^{2}$ & 4451.87 & 3088.17 & 683.00 \\
$\sum_{j} a_{j m}{ }_{j m} / \sum_{j} a^{2}{ }_{j 1}$ & 1.00 & 0.69 & 0.15
\end{tabular}

Table. 7

The Extracted Factors for the SUBJECT $\mathrm{H}$.

\begin{tabular}{c|rrr}
\hline & \multicolumn{1}{c}{$a_{1}$} & \multicolumn{1}{c}{$a_{2}$} & \multicolumn{1}{c}{$a_{3}$} \\
\hline 1 & 26.06 & -68.84 & -29.52 \\
2 & 20.39 & -58.64 & -20.78 \\
3 & 43.95 & -23.45 & -8.26 \\
4 & -39.75 & -41.31 & -10.41 \\
5 & 24.72 & 25.81 & 21.15 \\
6 & 65.04 & 58.71 & 12.44 \\
7 & -57.37 & 32.26 & 14.63 \\
8 & 28.47 & 66.42 & 24.97 \\
9 & -111.50 & 9.04 & -4.26 \\
$\sum_{j} a_{j m}^{2}$ & 25981.93 & 20081.08 & 2937.60 \\
$\sum a_{j m}^{2} / \Sigma a_{j 1}^{2}$ & 1.00 & 0.77 & 0.11 \\
$j$ & & &
\end{tabular}

Table. 6

The Extracted Factors for thF SUEJECT IN

\begin{tabular}{r|rrr}
\hline & \multicolumn{1}{|c}{$\boldsymbol{a}_{1}$} & \multicolumn{1}{c}{$\boldsymbol{a}_{2}$} & \multicolumn{1}{c}{$a_{3}$} \\
\hline 1 & 38.24 & 37.56 & -5.10 \\
2 & 34.08 & 27.67 & 9.07 \\
3 & 37.91 & 5.29 & 1.15 \\
4 & -8.58 & 27.60 & 5.55 \\
5 & 6.21 & -22.90 & 19.17 \\
6 & 18.58 & -47.62 & -24.26 \\
7 & -37.86 & -8.00 & 6.23 \\
8 & -21.15 & -34.26 & -1.13 \\
9 & -67.44 & 14.67 & -10.66 \\
$\sum_{j} a^{2}{ }_{j m}$ & 1094.7 .16 & 7211.16 & 1250.17 \\
$\sum_{j} a_{j m}{ }_{j m} \sum_{j} a^{2}{ }_{j 1}$ & 1.00 & 0.66 & 0.11
\end{tabular}

regarding the third factor may be summed up as follows :

a) When two factors were extracted, a considerable number of negatives began to appear in the main diagonal of the residual matrices. The secondfactor residual matrices are shown in Table 8 to 10 . Disregarding errors, elements in the main diagonal of these matrices cannot be negative because $b_{y j}^{2}$ is equal to $d^{2} y$, i. e., the square of 
Table. 8

The Second Factor Restdual Matrix for the Subject S.

\begin{tabular}{r|rrrrrrrrr}
\hline & \multicolumn{1}{|c}{1} & \multicolumn{1}{c}{2} & \multicolumn{1}{c}{3} & \multicolumn{1}{c}{4} & \multicolumn{1}{c}{5} & \multicolumn{1}{c}{6} & 7 & 8 & \multicolumn{1}{c}{9} \\
\hline 1 & 75.17 & -18.76 & 18.78 & -75.12 & -166.00 & 5.17 & -199.47 & 160.77 & 199.44 \\
2 & -18.76 & -140.20 & 88.86 & 70.52 & -10.45 & 57.52 & 1.38 & 4.32 & -53.18 \\
3 & 18.78 & 88.86 & -125.55 & 18.32 & 86.43 & 26.07 & -39.76 & -75.66 & 2.53 \\
4 & -75.12 & 70.52 & 18.32 & -13.18 & 89.71 & -89.18 & 237.36 & -89.69 & -148.80 \\
5 & -166.00 & -10.45 & 86.43 & 89.71 & -53.71 & -78.05 & 157.55 & 55.64 & -81.12 \\
6 & 5.17 & 57.52 & 26.07 & -89.18 & -78.05 & -26.77 & -191.42 & 21.35 & 275.25 \\
7 & -199.47 & 1.38 & -39.76 & 237.36 & 157.55 & -191.42 & -9.73 & 72.23 & -28.15 \\
8 & 160.77 & 4.32 & -75.66 & -89.69 & 55.64 & 21.35 & 72.23 & -5.67 & -143.22 \\
9 & 199.44 & -53.18 & 2.53 & -148.80 & -81.12 & 275.25 & -28.15 & -143.22 & -22.73
\end{tabular}

Table. 9

The Srcond Factor Residual Matrix for the Subject In.

\begin{tabular}{r|rrrrrrrrr}
\hline & \multicolumn{1}{|c}{1} & 2 & \multicolumn{1}{c}{3} & \multicolumn{1}{c}{4} & \multicolumn{1}{c}{5} & \multicolumn{1}{c}{6} & 7 & 8 & \multicolumn{1}{c}{9} \\
\hline 1 & 108.93 & 67.05 & -133.20 & -60.80 & -122.97 & 79.76 & 39.85 & 2.87 & 18.50 \\
2 & 67.05 & -86.06 & 68.38 & 197.04 & 191.49 & -240.53 & 2.61 & 46.70 & -246.61 \\
3 & -133.20 & 68.38 & -147.29 & 52.99 & 116.37 & 96.58 & -42.80 & -170.05 & 159.37 \\
4 & -60.80 & 197.04 & 52.99 & -560.24 & 359.57 & -548.37 & 178.71 & 10.04 & 370.72 \\
5 & -122.97 & 191.49 & 116.37 & 359.57 & -98.32 & -86.33 & 219.91 & -34.92 & -544.52 \\
6 & 79.76 & -240.53 & 96.58 & -548.37 & -86.33 & 150.68 & -219.33 & 155.42 & 612.82 \\
7 & 39.85 & 2.61 & -42.80 & 178.71 & 219.91 & -219.33 & -102.80 & 101.78 & -178.24 \\
8 & 2.87 & 46.70 & -170.05 & 10.04 & -34.92 & 155.42 & 101.78 & -221.14 & 109.45 \\
9 & 18.50 & -246.61 & 159.37 & 370.72 & -544.52 & 612.82 & -178.24 & 109.45 & -302.30
\end{tabular}

Table. 10

The Second Factor Resinual Matrix for the Subject H.

\begin{tabular}{r|rrrrrrrrr}
\hline & \multicolumn{1}{|c}{1} & \multicolumn{1}{c}{2} & \multicolumn{1}{c}{3} & \multicolumn{1}{c}{4} & \multicolumn{1}{c}{5} & \multicolumn{1}{c}{6} & 7 & 8 & \multicolumn{1}{c}{9} \\
\hline 1 & 2838.35 & 1709.54 & 490.54 & 1082.91 & -2207.94 & -1782.28 & -1935.22 & -1048.28 & 852.14 \\
2 & 1709.54 & 707.95 & 1008.62 & 1789.58 & -1133.57 & -981.44 & -1482.22 & -1310.76 & -307.86 \\
3 & 490.54 & 1008.62 & 280.97 & 305.07 & 606.15 & -893.82 & -170.79 & -1493.29 & -133.81 \\
4 & 1082.91 & 1789.58 & 305.07 & 495.41 & 439.68 & -1562.47 & 718.41 & -2054.32 & -1213.84 \\
5 & -2207.94 & -1133.57 & 606.15 & 439.68 & 996.16 & 600.21 & 2261.43 & 1137.32 & -2700.09 \\
6 & -1782.28 & -981.44 & -893.82 & -1562.47 & 600.21 & 2159.67 & -719.40 & 897.31 & 2281.57 \\
7 & -1935.22 & -1482.22 & -170.79 & 718.41 & 2261.43 & -719.40 & -122.18 & 2046.33 & -595.76 \\
8 & -1048.28 & -1310.76 & -1493.29 & -2054.32 & 1137.32 & 897.31 & 2046.33 & 1816.58 & 8.46 \\
9 & 852.14 & -307.86 & -133.81 & -1213.84 & -2700.09 & 2281.57 & -595.76 & 8.46 & 1810.33.
\end{tabular}


the length of a vector from the centroid of the points to a joint $j$.

b) The configuration of the points, $A=a_{j_{1}}, a_{j_{2}}$, gave a highly reasonable result (vide infra).

c) The contribution of the additional factor in accounting for $B^{*}$ was relatively small. The contribution of each column of the matrix $A, \sum_{j} a^{2}{ }_{j m}$ and the ratio $\underset{j}{\Sigma} a_{j m}^{2} / \sum_{j} a_{j_{1}}$ were listed below the Tables. The contribution of the third centroid factor was about a tenth of that of the first centroid factor in each subject.

d) Applying the criterion given by Torgerson, we came to the conclusion that the matrices $B^{*}$ were in general of rank 2 except for variable errors. The criterion was as follows: If the matrix $B^{*}$ is of rank 2 , then the equaion :

$$
\begin{aligned}
\sum_{j k} \sum b_{j k}^{*}=\left(\underset{j}{\Sigma} a_{j_{1}}^{2}\right)^{2} & +\left(\underset{j}{\Sigma} a^{2}{ }_{j 2}\right)^{2} \\
& \left.+2 \underset{j}{\sum_{j 1}} a_{j 2}\right)^{2}
\end{aligned}
$$

should hold. To be exact, $a_{j_{1}}$ and $a_{J_{2}}$ in the equation must be the values obtained by the method of principal axes. It can be assumed, however, that if $a_{j_{1}}$ and $a_{j_{2}}$ were replaced by the centroid values, the right-hand term of the equation would give substantially the same values. Difference, if any, would be such as to decrease the value of the right-hand term. Torgerson obtained in his analysis $15.24 \times 10^{2}$ for the lefthand term and $14.96 \times 10^{2}$ for the right putting the centroid values into $a_{j_{1}}$ and $a_{f_{2}}$. These values, he admitted, are sufficiently close to warrant the hypo- thesis that the matrix $B^{*}$ is of rank.2. In the present case, these values were :

$\begin{array}{ccc}\text { Subject } & \begin{array}{c}\text { The left-hand } \\ \text { term }\end{array} & \begin{array}{c}\text { The right-han } \\ \text { term }\end{array} \\ \text { S } & 29.11 \times 10^{6} & 29.36 \times 10^{6} \\ \text { In } & 176.79 \times 10^{4} & 171.84 \times 10^{6} \\ \text { H } & 15.88 \times 10^{8} & 10.79 \times 10^{8} .\end{array}$

In comparing these corresponding values one should keep in mine the fact that $b^{*}{ }_{j i}$ was defined on a scale in the present case entirely different from that of Torgerson. The means of the absolute values of $b_{j k}$ were, 3.22 in the case of Torgerson and in the present case $527.41,1253.40$ and 3850.01 for the subject $\mathrm{S}$, In and $\mathrm{H}$ respectively. The relative sizes of the unit will be, therefore; $1.59 \times 10^{2}$ in the subject $S, 3.77 \times 10^{2}$ in the subject In and $1.16 \times 10^{3}$ in the subject $H$ if Torgerson's unit is taken to be 1 . If values of both terms in Torgerson are multiplied by the square of these coefficients (since the unit is squared in the equation (9)), then we have:

$\begin{array}{ccc}\text { Subject } & \begin{array}{c}\text { The left-hand } \\ \text { term }\end{array} & \begin{array}{c}\text { The right-hand } \\ \text { term }\end{array} \\ \text { S } & 38.56 \times 10^{6} & 37.85 \times 10^{6} \\ \text { In } & 216.56 \times 10^{6} & 212.58 \times 10^{6} \\ \text { H } & 20.57 \times 10^{9} & 20.20 \times 10^{9} .\end{array}$

Comparing these two tables it may be concluded that goodness of agreement between the right-and the left-hand values is the same or better than that of Torgerson's for the subjects In and $\mathrm{S}$ and slightly worse for the subject $\mathrm{H}$. Plotting $A=\left\|a_{J_{1}}, a_{j_{2}}\right\|$ on the plane, two new axes were easily determined, which were unequivocally oriented in 
directions comparable to the value and chroma dimensions of the Munsell system. The rotation of the axes followed. Table 11 to 13 give the matrices after the rotation, $V=\left\|v_{j_{1}}, v_{j_{2}}\right\|$, in which the first axis corresponds to value of the color $j$ and $v_{j_{2}}$ against the Munsell chroma, the series of points lay clearly in a straight line for each subject. The results for the subject In are shown in Figure 2 and 3 as examples. Straight lines were fitted by

Table. 11

The Matrix atter the Rotation Represhenting the Scaled Confrguration of the Colors for the SUbJect S.

\begin{tabular}{r|rrrrrrrrr}
\hline & \multicolumn{1}{c}{1} & \multicolumn{1}{c}{2} & \multicolumn{1}{c}{3} & \multicolumn{1}{c}{4} & \multicolumn{1}{c}{5} & \multicolumn{1}{c}{6} & \multicolumn{1}{c}{7} & \multicolumn{1}{c}{8} & \multicolumn{1}{c}{9} \\
\hline$v_{1}$ & 26.94 & 23.97 & 22.16 & 1.10 & 0.73 & 1.83 & -24.21 & -15.90 & -36.62 \\
$v_{2}$ & -22.53 & -17.30 & 5.17 & -23.82 & 18.58 & 30.35 & 3.51 & 23.53 & -18.02
\end{tabular}

Table. 12

The Matrix aftrer the Rotation Rlypreshating the Scalej, Configuration of the CoLors for the SUbJict IN.

\begin{tabular}{r|rrrrrrrrr}
\hline & 1 & 2 & 3 & 4 & 5 & \multicolumn{1}{c}{6} & \multicolumn{1}{c}{7} & 8 & \multicolumn{1}{c}{9} \\
\hline$v_{1}$ & 48.78 & 41.49 & 37.43 & 1.38 & -1.99 & 1.18 & -38.31 & 31.60 & -58.35 \\
$v_{2}$ & -22.22 & -14.35 & 7.99 & -28.87 & 23.69 & 51.08 & -5.43 & 24.96 & -36.85
\end{tabular}

Table. 13

The Matrix after the Rotation Represinting the Scaled Configuration of the Colors rok the Subject $H$.

\begin{tabular}{r|rrrrrrrrr}
\hline & \multicolumn{1}{c}{1} & \multicolumn{1}{c}{2} & 3 & \multicolumn{1}{c}{4} & \multicolumn{1}{c}{5} & \multicolumn{1}{c}{6} & \multicolumn{1}{c}{7} & \multicolumn{1}{c}{8} & \multicolumn{1}{c}{9} \\
\hline$v_{1}$ & 67.62 & 56.34 & 47.40 & 2.10 & -1.39 & 2.95 & -63.06 & -28.00 & -83.96 \\
$v_{2}$ & -29.08 & -26.07 & 15.32 & -57.29 & 35.71 & 87.57 & -18.86 & 66.62 & -73.92
\end{tabular}

value and the second to chroma and the origin of both axes are located in the centroid of the colors. By the matrix $V$, the configuration of colors in the value-chroma plane was finally determined, $v_{J_{1}}$ giving the position of a color $j$ along the value dimension and $v_{j_{2}}$ along the chroma dimension. When $V_{J_{1}}$ was plotted against the Munsell the least square method as follows:

$$
\begin{array}{ccc}
\text { Subject } & \begin{array}{c}
X_{1}: \text { Munsell } \\
\text { value. }
\end{array} & \begin{array}{c}
X_{2} \text { : Munsell } \\
\text { chroma. }
\end{array} \\
\text { S } & V_{1}=1822 X_{1}-93.72 & V_{2}=6.79 X_{2}-47.50 \\
\text { In } & V_{1}=31.07 X_{1}=159.84 & V_{2}=9.94 X_{2}-72.42 \\
\text { H } & V_{1}=42.66 X_{1}-219.44 & V_{2}=18.47 X_{2}-134.64
\end{array}
$$

By means of these equations the Munsell system can be superimposed upon the diagram representing the matrix $V$. Figure 4 to 6 give these 


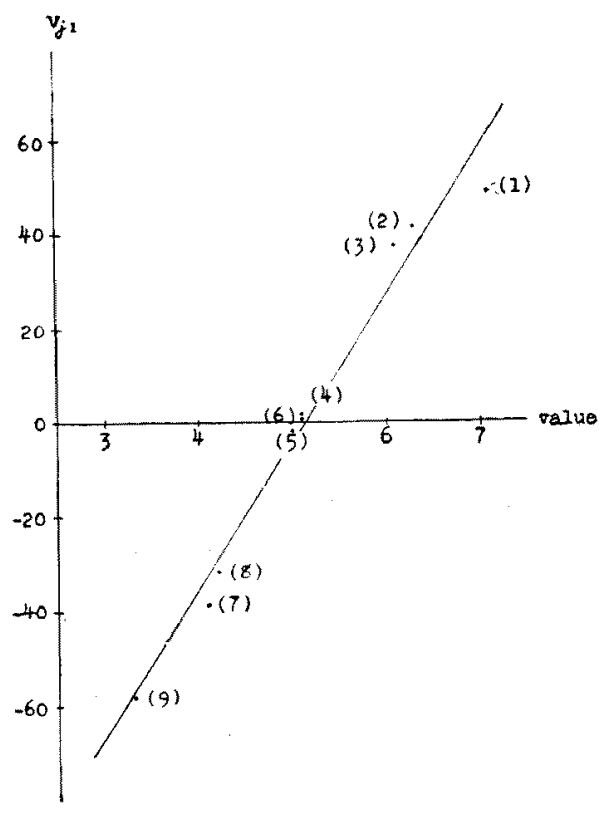

Fig. 2 Subject : In. Mensell Value with $v_{1}, i . e_{2}$, the Scalmo Position along thes Value Dimension.

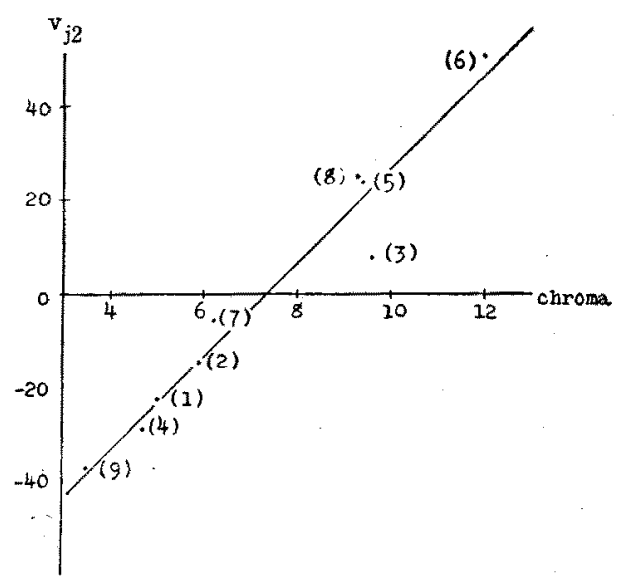

Fig.3. Subject : Iv. Munsfll Chroma with $v_{2}$, i.e., THE:SCALEd Posttion ALONG THE CmRom Dinension.

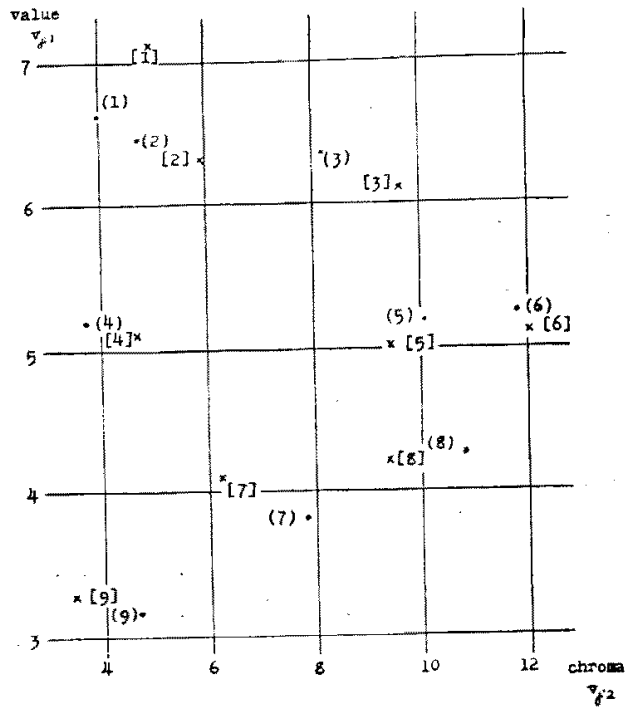

Fig. 4. Subject : S. The Dots Repensent the Sualis Configuration of the Colors and Crosses the Positions in the Munseiti System

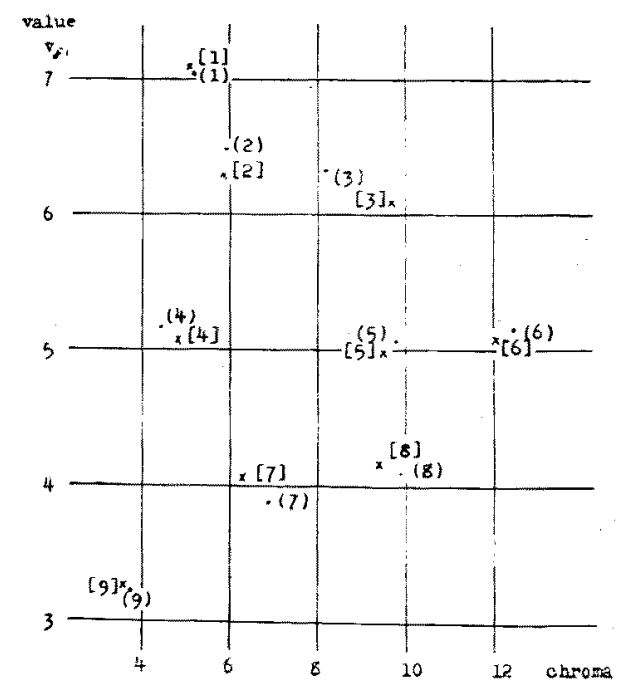

Fig. 5. Subject : In. The Dots RepreSENT the SCaled Configuration of the Colors and the Crosses the Positions in the Monsell SYSTEM. 
diagrams where dots represent the configuration of the colors determined by $V$ and the crosses positions in the Munsell system. The horizontal and vertical lines in the Figures indicate the positions of the corresponding Munsell value and chroma superimposed upon the plane;

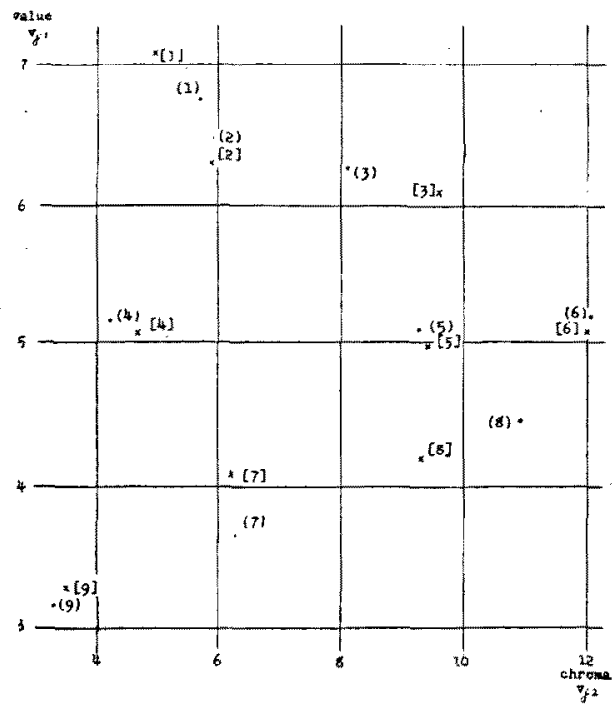

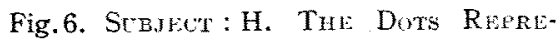
SENT the Scaled Conflgltation of THF Colors aND THE Crossms the Positions ix the Musiselt Srster.

the real co-ordinates of the plane, namely the first and the second axes in $V$ are omitted. In each case, the crosses and the dots are in good agreement. Besides, if the matrices $V$ of three subjects are plotted in a plane by adjusting individual differences in the metric in an appropriate way, three configurations are also in good agreement as shown in Figure 7. It is quite remarkable that deviations are more or less in the same direction in all subjects.
Individual differences are not qualitative, but quantitative with the three subjects.

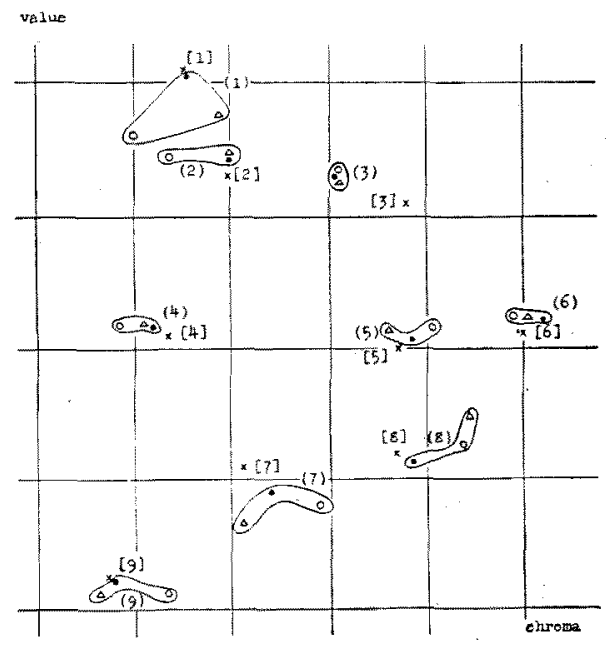

Fig.7. The Crosses Ruplesent the PoSTTONS OF THE COLORS IN THE Mussell System and $\bigcirc$ The Scalen Comfguratron For thy Sitrjigr $S$, ror the SubJect IN ANI $\triangle$ FOR THE SUBJECT H.

Two axes, the ordinate representing value and the abscissa representing chroma, were orthogonal in all subjects. It might be well to mention that the orthogonality was preserved in the rotation and not enforced. It was determined by the data themselves. The most important characteristic of the configuration determined by $V$ and plotted in Figure 4 to 6 is that the distance between any two colors in the plane, whatever direction it may take, is proportional to the degree of perceptual difference between these colors for each subject. And it is noteworthy that in such a plane the unit of the Munsell value appeard considerably larger than 
that of the Munsell chroma even if the circumstances were taken into consideration that in the Munsell notation two chroma steps were generally defined to represent approximately a sense distance equivalent to one value step. That means, variation in the Munsell value contributes more to the impression of difference than that in Munsell chroma if variation in both dimensions are taken into consideration. There was no exception among three subjects. The same fact also appeared in the data of Torgerson though he did not mention it. Furthermore, the fact that the agreement between the experimental results and the positions of the colors in the Munsell system was in general closer in the direction of brightness than in the direction of saturation may not be overlooked. In other words, the goodness of fit of the above mentioned equation $V_{1}=a X_{1}-b$ is more or less better than that of the equation $V_{2}=a X_{2}-b$ for all subjects. Concerning the subject In, the difference will be apparent in Figure 2 and 3 . Though slight, the difference of the same kind can be observed in the data of Torgerson. This finding obtained in the final results of the analysis seems to be in line with the introspective reports of the subject during the observation that the difference in brightness is phenomenally more distinct than that in saturation when the subject casts his first glance at the pair of the colors presented.
In general, it may be said that each of the three subjects perceived the relation between two red colors differing in brightness and saturation in a way similar to that represented in the value-chroma plane of the Munsell system, but variation in the Munsell value seemed to contribute more to the impression of difference than defined in the Munsell notation. And variation in brightness was phenomenally more distinct than that in saturation. These findings applied equally to all the subjects. They were in harmony with the results of Torgerson who worked with the pooled data of a number of subjects. Regarding his assumptions for pooling the data the mention was made in the previous section.

\section{SUMMARY}

1. The method of multi-dimensional scaling developed by Torgerson was applied to analysis of perceptual similarity or difference among colors.

2 . In contrast to the study of Torgerson in which he analyzed the pooled data of 40 subject's judgements and developed a common color space, we tried to obtain separate result for each subject by the use of the multi-dimensional scaling for individual measurement.

3. Nine colored papers were chosen as stimuli which were of the same red but differed from each other in brightness and saturation. The particular hue used in this experiment was in the 
vicinity of $5 R$, the values approximately from 3 to 7 and the chromas approximately from 4 to 12 in the Munsell notation.

4. Three subjects took part in the experiment which was carried out individually. A triad of colors, $i, j$ and $k$ was presented in a row in which $k$ was in the middle of $i$ and $j$. The subject was asked to express the degree of difference in similarity between $i$ and $k$ and between $j$ and $k$ by displacing $k$ either toward $i$ or $j$ from its original position. An analytical judgement was not required but the subject was instructed to rely upon general impression of similarity.

5. By the method of multi-dimensional scaling, it was possible to develop for each subject two-dimensional Euclidean color space where colors were arranged in such a way that distance between any two colors, whatever direction it took, was proportional to the degree of perceptual difference of these colors.

6. In this space two orthogonal axes were identified which corresponded to brightness and to saturation respectively.

7. The configuration of the colors in this space as calculated from the experimental data was in good agreement with the positions of the colors in the Munsell system which was superimposed upon this space.

8. For all subjects, however, varia tion in the Munsell value contributed more to the impression of similarity or difference than defined in the Munsell notation.

9. The agreement between the configuration of the colors and that part of the Munsell system was in general closer in the direction of brightness than in the direction of saturation due presumably to the fact that variation in brightness was phenomenally more distinct than that in saturation.

10. Results of the three subjects excellently agreed with one another and they were also in harmony with what Torgerson obtained with the pooled data of 40 subjects.

\section{REFERENCES}

1) Torgerson, W. S.: Multi-dimensional scaling theory and method. Phychometrika, $1952,17,401-419$.

2) Torgerson, W. S.: A theoretical and empirical investigation of multi-dimensional scaling. Research Bull., RS-51-14, Educational Testing Service, 1951.

3) Reese, T.W.: The application of the theory of physical measurement to the measurement of psychological examples. Psychol. Monogr., 1943, 55, No.3.

4) Boring, E.G. : The Psychophysics of color tolerance. Amer. J. Psychol., 1939, 52, 384-394.

5) Stevens, S.S. : A scale for the measurement of a psychological magnitude : loudness. Psychol.Rev., 1936, 43, 4.05-416.

6) Stevens, S.S. and Volkmann, J.: The relation of pitch to frequency: a revised scale. Amer. J. Psychol., 1940, 53, 329-353.

7) Judd, D. B. : Colors in business, science, and industry. New York, John Wiley, 1952.

8) Burnham, R.W. : Comparison of color systems with respect to uniform visual spacing. J. opt. Soc. Amer., 1949, 39, 387-392. 
9) Newhall, S.M.: The ratio method in the review of the Munsell colors. Amer. J. Psychol., 1939, 52, 394-405.

10) Balinkin, I.A. : Industrial color tolerances. Amer. J. Psychol., 1939, 52,428-448.

11) Mosteller, F. : Remarks on the method of paired comparisons : 1 . The least squared solution assuming equal standard deviations and equal correlations. Psychometrika, $1951,16,3-9$.

12) Messick, S. J. and Abelson, R. P. : The additive constant problem in multi-dimen- sional scaling. Research Bull., RS-54-13, Educational Testing Service, 1954 .

13) Messick, S. J. : The perception of attitudes relationships: A multi-dimensional scaling approach to the structure of social attitudes. Research Bull., RB-5A-27, Educational Testing Service, 1954.

14) Young, G. and Householder, A.S. : Discussion of a set of points in terms of their mutual distances. Psychometrika, 1938, 3, $19-22$. 